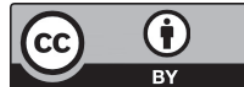

https://creativecommons.org/licenses/by/4.0/

\title{
LA GESTIÓN DEL CAPITAL INTELECTUAL EN UNIVERSIDADES: UNA REVISIÓN DE LA LITERATURA
}

\section{The management of intellectual capital in universities: A review of the literature}

CARLOS MARIO DURANGO YEPES ${ }^{1}$

Recibido:29 de agosto de 2018. Aceptado:24 de octubre de 2018

DOI: http://dx.doi.org/10.21017/rimci.2019.v6.n11.a62

\begin{abstract}
Resumen
El propósito de este trabajo es revisar la literatura investigativa existente sobre los informes de capital intelectual (Cl) en las universidades, para establecer una mejor comprensión de cómo las universidades del mundo han respondido al reto de divulgar su Cl. Para lo cual se seleccionaron y evaluaron los artículos relacionados con el tema, articulándolos de acuerdo con los hallazgos mismos de la literatura. El artículo realiza tres contribuciones específicas. La primera, es proporcionar una visión global de los avances en las universidades que han implementado metodologías y divulgado informes de $\mathrm{Cl}$ en lo que va de este siglo. La segunda, suministrar información sobre los modelos de $\mathrm{Cl}$ que han elegido las universidades como sistema de gestión de intangibles. La tercera, es proporcionar información metodológica de aquellos trabajos de investigación más representativos sobre divulgación del $\mathrm{Cl}$.
\end{abstract}

Palabras clave: Informes de capital intelectual, Universidades, Revisión de la literatura.

Clasificación JEL: 123, 128, M10, M41

\begin{abstract}
The purpose of this paper is to review the existing research literature on intellectual capital (IC) in universities, to establish a better understanding of how the world's universities have responded to the challenge disclose your IC. For which they were selected and assessed articles related to the topic, articulating according finds himself literature. The paper makes three specific contributions. The first contribution is to provide an overview of developments in the universities that have implemented methodologies and IC reports released so far this century. Second, information on IC models that have chosen universities as intangibles management system. The third is to provide methodological information to those most representative works of research on disclosure of IC.
\end{abstract}

Keywords: Intellectual capital reporting, Universities, Literature review.

\section{INTRODUCCIÓN}

A Unque la literatura científica y profesional ha proporcionado numerosos estudios acerca de los informes de Capital Intelectual (CI) en las empresas, se requiere más investigación empírica para las universidades. Estudios como el de Ramirez et al.[1] confirman que para mejorar la información contenida en los informes anuales de las universidades, se requiere ampliar la información proporcionada por los estados contables actuales. Este trabajo examina la propuesta de que la gestión del conocimiento es una estrategia importante para las universidades, y que esto se reflejará a través de la

1 Magister en Gestión Tecnológica de la Universidad Pontificia Bolivariana. Especialista en Asesoria y Consultoria de Organizaciones e Ingeniero Químico de la Universidad de Antioquia. Profesor investigador en temas de Gestión del Conocimiento, Gestión de la Innovación y Gestión Tecnológica en especializaciones y en la Maestría en Administración. Actualmente Jefe Oficina de Laboratorio de la Fábrica de Licores y Alcoholes de Antioquia. http://orcid.org/0000-0003-2695-4200. Correo electrónico: carlosdster@gmail.com 
divulgación de los ítems de capital intelectual en el informe anual de la universidad. Como apoyo a esta expectativa, se hace evidente que es Europa, sobre todo a principios de este siglo, la pionera en la divulgación de informes de activos intangibles, o de informes de capital intelectual (CI) a nivel empresarial y universitario. El objetivo de este artículo es revisar la literatura investigativa existente sobre los informes de capital intelectual en las universidades, para establecer una mejor comprensión de cómo las universidades del mundo han respondido al reto de divulgar su capital intelectual.

El proceso de revisión de los artículos se llevó a cabo en dos etapas diferentes. El conjunto de datos se limita a un período de quince años desde 2000 hasta 2014. Es decir, desde el 2000 hasta lo que va del 2014. La primera etapa consistió en la selección de revistas. Estas comprenden ocho revistas de gestión y economía reconocidas internacionalmente y cuatro revistas como, Journal of Intellectual Capital, British Accounting Review, Accounting, Auditing \& Accountability Journal y Accounting Forum. Las ocho revistas de Gestión y Economía fueron seleccionadas porque están comprometidas con la publicación de la investigación interdisciplinaria y han publicado en el área de capital intelectual[2].

En la segunda etapa, se examinaron 83 títulos y resúmenes de todos los artículos publicados en las revistas durante el período que contienen las palabras Informes de CI, mientras que se eliminan los artículos que no estén relacionados con los Informes de CI en universidades. Como resultado se identificaron 52 artículos que se centran en informes de capital intelectual en universidades. Luego, se descargan los artículos en versión PDF y se almacenan en una base de datos endnote referenciando detalles.

En la tercera etapa, se elaboró un mapa conceptual con los aspectos evolutivos del concepto de capital intelectual, los métodos utilizados, los efectos de la gestión del capital intelectual y un diagnóstico de las investigaciones de CI en las universidades.

Como resultado el contenido de este artículo está organizado en cinco secciones: la sección dos, aporta una visión histórica del concepto de capital intelectual; la sección tres, presenta una breve reseña de los métodos de medición del capital inte- lectual, enfatizando en el cuadro devmando integral por su pertinencia en las universidades; la sección cuatro, muestra los efectos de la gestión del capital intelectual en las universidades; la sección cinco, aporta una revisión de lo que se ha investigado sobre los informes de capital intelectual en las universidades del mundo. Finalmente, se presentan las conclusiones de la revisión de la literatura investigativa sobre capital intelectual en las universidades.

\section{El desarrollo del concepto DE CAPITAL INTELECTUAL}

El conocimiento tiene una historia sorprendentemente larga, fundado en la meso-economía del primer tercio del siglo XX, que luego se desarrolló en la segunda mitad en el punto de vista micro-económico (basado en la firma) Chamberlin[3] y más tarde Penrose[4] fueron los contribuyentes de este primer trabajo. La obra de Schumpeter de 1912[5] precede este trabajo y ve el uso de las nuevas combinaciones de recursos por parte de empresarios como la base del crecimiento económico cíclico. Sin embargo, la perspectiva de Schumpeter era centrada en lo macroeconómico y la invención, a diferencia de la innovación, se trató como una variable exógena a la empresa. El examen de las contribuciones de Robinson[6] y Chamberlin[3] muestra cómo los conceptos que ellos desarrollaron han sobrevivido hasta el presente. Por ejemplo, Chamberlin[3] identificó que algunas de las capacidades clave de las empresas como el saber hacer técnico, la reputación, patentes, marcas registradas, capacidad de los administradores para trabajar en equipo, muchas de ellas son comunes en la literatura reciente de marketing y estrategia[7][8]. Muchos de los trabajos de Edith Penrose[4] citados sobre la teoría de crecimiento de la empresa, rechazan la visión que una empresa es solo una unidad administrativa, en cambio, la vieron como recursos productivos a disposición de los administradores. Ella sugirió que una empresa se mide mejor por alguna medida de los recursos productivos utilizados. Esto llevó directamente al desarrollo de las ideas relativas a la ventaja competitiva en el último tercio del siglo XX.

El trabajo de Penrose[4] proporciona una orientación adicional para el desarrollo del capital in- 
telectual como un enfoque para la gestión empresarial. Por ejemplo, la definición clara de lo que un recurso puede ser y cómo se diferencia de las actividades y servicios es crucial. Esto condujo a la noción de que los servicios producidos por los recursos dependen de los recursos que se utilizan. Un recurso dado se puede utilizar en diferentes combinaciones con otros recursos para dar diferentes servicios o generar una variedad de otros recursos. Por otra parte, el desarrollo de una empresa se ve limitada hasta cierto punto por la naturaleza y cualidades de los recursos que posea actualmente. Este pensamiento llevó a otros autores a considerar el desarrollo y el despliegue de recursos[9]; [10];[11];[12][13] y la relación entre los recursos y el alcance de la empresa[14];[15]; $[16] ;[17]$.

El término $\mathrm{CI}$, cuando hace referencia a una universidad, es un término que se utiliza para cubrir todos los activos no tangibles o no físicos de la institución, incluyendo los procesos, la capacidad de innovación, las patentes, el conocimiento tácito de sus miembros y sus habilidades y talentos, el reconocimiento de la sociedad, su red de colaboradores y contactos, entre otros. El CI es la colección de los intangibles que «permite a una organización transferir una colección de recursos humanos, financieros y materiales en un sistema capaz de crear valor para los grupos de interés»[18]. Los componentes del CI de una universidad se han clasificado de diversas maneras. No obstante, la clasificación tripartita es, sin duda, la más aceptada en la literatura como (Leitner[19]; Elena[20]; Ramírez et al.[21]; Cañibano y Sánchez[22]; Sánchez et al.[23]; Bezhani[24]; Bodnar et al.[25]: Casanueva y Gallego[26]; Secundo et al.[27]. No obstante, Bueno et al.[28] introducen un cuarto componente, el capital emprendimiento e innovación. El Capital Intelectual es formado por cuatro componentes básicos y estrechamente interrelacionados:

\section{A. El capital humano}

Es la suma del conocimiento explícito y tácito de las personas de la universidad (profesores, investigadores, administradores, personal de administración y servicios), adquirido a través de la educación formal y no formal y los procesos de actualización incluidos en sus actividades.

\section{B. El capital estructural}

Es el conocimiento explícito en relación con los procesos internos de difusión, comunicación y gestión del conocimiento científico y técnico en la universidad. El capital estructural se puede dividir en:

1) El capital organizacional: está compuesto por el entorno operativo derivado de la interacción entre investigación, gestión y organización de los procesos, rutinas organizativas, cultura corporativa y valores, procedimientos internos, y el sistema de información, entre otros.

2) El capital tecnológico[28]: se compone de cuatro elementos básicos: esfuerzo en I+D (gasto, personal y proyectos de I+D), dotación tecnológica (compra de tecnología como patentes y licencias, dotación de tecnologías de la producción, dotación de TIC), propiedad intelectual (patentes, marcas, licencias, dominios en internet) y procesos de inteligencia organizativa (información sobre patentes, conocimiento de la actividad de I+D de la competencia, información sobre líneas de investigación y tecnologías emergentes, conocimiento de posibles asociaciones con empresas para I+D).

\section{El capital relacional}

Puede ser definido como el conjunto de conocimientos que se incorporan a la organización y a las personas que la integran como consecuencia del valor derivado del número y calidad de las relaciones que de forma continuada se mantienen con los diferentes agentes del mercado y con la sociedad en general. Está integrado, en consecuencia, por el capital negocio y por el capital social. El capital negocio se compone de seis elementos básicos de relaciones con: clientes, proveedores, accionistas e inversores, aliados, competidores, instituciones de promoción y mejoramiento de la calidad[28]. El capital social, se refiere al valor que representa para la organización las relaciones que ésta mantiene con los restantes agentes sociales que actúan en su entorno, social y territorial, expresado en términos del nivel de integración, compromiso, cooperación, cohesión, conexión y responsabilidad social que quiere establecer con la sociedad[28]. 


\section{Capital de emprendimiento e innovación}

Se compone de actitud y capacidad de emprendimiento y de resultados de innovación expresados mediante: valores y actitudes de innovación, innovación en gestión, innovación internacional, innovación de servicio, innovación de procesos, innovación de modelo de negocio, innovación social, responsabilidad social corporativa.

\section{Modelos PARA LA MEDICIÓN DEL CI}

Durante las últimas décadas, el interés en el manejo del CI ha llevado al desarrollo de varios modelos para medirlo. Algunos de estos métodos fueron los intentos realizados por diferentes empresas para su uso interno y no el desarrollo de un modelo de medición universal. Existen diferentes enfoques para la clasificación de los modelos de medición. Sveiby, con base en las obras de Luthy[29], y Williams[30], clasifica los enfoques existentes en cuatro clases: 1) Los modelos directos de CI (DIC) que estiman el valor de los activos intangibles mediante la identificación de sus componentes; 2) Modelos de capitalización de mercado (MCM) que calculan la diferencia entre la capitalización de mercado de una empresa y su capital contable con el fin de determinar el valor de sus activos de CI o intangibles; 3) El Retorno sobre los Activos (ROA) - el resultado es la rentabilidad sobre los activos de la empresa que luego se compara con la media del sector; 4) Modelos Scorecard (SC) que identifican los distintos componentes del CI intangible, indicadores e índices que se generan y se informan los cuadros de mandos o en forma de gráficos . Dada la reciente proliferación de los modelos de medición, es conveniente establecer una visión general de ellos. (Ver tabla I).

Todos los modelos para la medición de CI son dependientes del contexto y se demuestra que es muy difícil trazar fronteras claras entre los distintos elementos medibles sin superposición (Jurczak[32]; Lev, B. and Zambon, S.[33]; Mouritsen[34]. Cualquiera que sea el modelo que se elija, es importante que la organización sea constante en el uso de la solución adecuada. Para desarrollar un análisis dinámico del CI y comparar los resultados de un año a otro, no se debe cambiar el método de manera frecuente.
Un enfoque innovador que puede enriquecer el control de la gestión de los intangibles de las universidades, y que se ubica en las técnicas ScoreCard, es el cuadro de mando integralv(CMI), el cual presenta un enfoque integrador que complementa la información proporcionada por las medidas financieras tradicionales con tres perspectivas adicionales (clientes, procesos internos y aprendizaje y crecimiento), las cuales permiten controlar el proceso de construcción de capacidades y adquisición de recursos intangibles necesarios para el crecimiento futuro.

La elección del Cuadro de Mando Integral como sistema de gestión de intangibles en las universidades se debe a que, hoy por hoy, es considerado como una de las herramientas más eficaces para implantar y hacer operativa la cultura de la calidad y el incremento del rendimiento (véase, por ejemplo, Pupius et al.[35]; Johnson[36] Cullen et al.[37] y Rohm[38]), y a que ya ha sido implementada con éxito en algunas universidades (por ejemplo, University of Edinburg, the Open University, Glasgow Caledonian University, Napier University, University of California[39][40][41][42].

La aplicación del CMI en las universidades, al igual que en el resto de organizaciones, se deriva y construye partiendo de la visión y estrategia de la institución y está compuesto por un conjunto de indicadores financieros y no financieros relativos a los factores que determinan su éxito. En la elaboración de un CMI es necesario que la estrategia esté definida[43]. Con este fin, un importante paso de inicio es el desarrollo de un Plan Estratégico institucional que recoja los objetivos y estrategias a largo plazo, así como las líneas de trabajo. La construcción del modelo se hace de tal forma que los indicadores diseñados para cada caso particular, mantengan entre ellos una fuerte conexión, planteada en forma de relaciones de causa-efecto a lo largo de la cadena de valor objeto de análisis. Tales indicadores pueden ser de tipo cualitativo (encuestas de opinión realizadas al personal docente e investigador, al personal de administración, a los alumnos, a las empresas, etc.) o cuantitativo, pudiendo ser éstos monetarios (como son el coste de un programa por alumno o por crédito docente, complemento medio de docencia por profesor) o no monetarios (porcentaje de eficiencia terminal por programa, tiempo medio por alumno en finalizar una titulación, etc.). 
Tabla I. Breve reseña de los modelos de medición del Cl.

\begin{tabular}{|c|c|c|c|}
\hline Modelo & Tipo & Fortalezas & Debilidades \\
\hline DIC & Monetario & $\begin{array}{l}\text {-Permite la medición separada de los } \\
\text { componentes del CI. } \\
\text {-Proporciona una visión global de una } \\
\text { organización de riqueza intelectual. } \\
\text {-Las mediciones se basan en eventos. }\end{array}$ & $\begin{array}{l}\text {-Específico de una categoría particular de } \\
\text { organizaciones. } \\
\text {-No es apropiado para la evaluación } \\
\text { comparativa o comparaciones. } \\
\text {-Número limitado de componentes. }\end{array}$ \\
\hline MCM & Monetario & $\begin{array}{l}\text {-Permite la comparación de las } \\
\text { organizaciones en un campo particular } \\
\text {-Proporciona un valor monetario del CI. } \\
\text {-Apropiada para la referenciación } \\
\text { competitiva y las comparaciones }\end{array}$ & $\begin{array}{l}\text {-No es adecuado para una visión general } \\
\text { del desarrollo. } \\
\text {-Su enfoque puramente económico es } \\
\text { limitante. }\end{array}$ \\
\hline $\mathrm{ROA}$ & Monetario & $\begin{array}{l}\text {-Apropiado para la referenciación } \\
\text { competitiva. } \\
\text {-Adecuado para comparar } \\
\text { organizaciones del mismo sector. } \\
\text {-Basado en las reglas contables } \\
\text { tradicionales. }\end{array}$ & $\begin{array}{l}\text {-Se caracteriza por la falta de información } \\
\text { que constituye CI. } \\
\text {-Su enfoque puramente económico es } \\
\text { limitante. }\end{array}$ \\
\hline SC & $\begin{array}{c}\text { No } \\
\text { monetario }\end{array}$ & $\begin{array}{l}\text { Proporciona un examen exhaustivo del } \\
\text { CI y del desempeño de los métodos } \\
\text { basados en la medición monetaria. }\end{array}$ & $\begin{array}{l}\text {-Sensible a los cambios del contexto. } \\
\text { - La cantidad de la información resultante } \\
\text { puede ser difícil de analizar; es difícil } \\
\text { obtener un resultado numérico. }\end{array}$ \\
\hline
\end{tabular}

Fuente: Gogan and Draghici [31].

Una vez definido el conjunto de indicadores para cada perspectiva, éstos se registran con valores históricos, se comparan con una meta o indicador objetivo y con el valor que resulte de las mejores prácticas (benchmarking).

Para adecuar el CMI al ámbito de las universidades, O'Neil y Harold[40] realizaron una serie de modificaciones: pasar del "balanced scorecard" a un "academia scorecard", rediseñando dos perspectivas para adaptarlas a la Universidad. La " perspectiva financiera" fue cambiada por " perspectiva de la administración académica", modificando la pregunta: "cómo

nos ven nuestros accionistas" por: "cómo nos ven los líderes universitarios". La original "perspectiva del cliente" fue cambiada por la "perspectiva de los dueños o sostenedores de la Universidad", identificando a estudiantes y a empleadores de los egresados, algún organismo público en el caso de las universidades estatales, como los mayores sostenedores (stakeholders). Para las otras perspectivas se mantuvieron los nombres (procesos internos y aprendizaje y crecimiento).

\section{Los EFECTOS de la Gestión Del Capital InTelectual EN LAS UNIVERSIDADES}

Según Cañibano y Sánchez[22], algunas de las preocupaciones principales de las empresas y de las universidades están relacionadas con los efectos de la gestión del capital intelectual: 1) el proceso de creación de valor, 2) en las demandas sociales de mayor responsabilidad, 3) en sus relaciones con los socios y aliados externos y, 4), el efecto de la divulgación del CI en los mercados de capital.

\section{A. Los procesos de creación de valor}

Es claro que la presentación y gestión de informes CI tienen sentido para las organizaciones si el ejercicio crea valor para los grupos de interés, y esto sucede si aumenta el beneficio, el valor de mercado o ambos[22].

Aunque existe un consenso general sobre la necesidad de nuevas técnicas de medición, hay obstáculos importantes en la medición de conocimientos por varias razones diferentes: una parte 
importante del conocimiento es implícito; los elementos diferentes del conocimiento son heterogéneos; el conocimiento no es observable, etc.[44].

Calcular el valor del capital intelectual es más complicado y arriesgado que el valor del capital tangible y aunque está claro que las empresas no invertirán en intangibles (tales como I + D) a no ser que ellos perciban que vale la pena, no es fácil la cuantificación del valor de los intangibles y los rendimientos que estos generan[45]. Aunque en general se acepta que los intangibles crean valor agregado, esta cadena de causa-efecto aún no se ha cuantificado[46].

Sin embargo, medir los intangibles es una cuestión muy importante, por varias razones. En primer lugar, es extremadamente difícil administrar los activos que no se pueden medir o determinar el éxito o el fracaso en las actividades de gestión de activos. Además, si los intangibles no se miden correctamente, una organización podría parecer estar actuando pobremente cuando de hecho está simplemente invirtiendo en intangibles[45].

En conclusión, es difícil establecer una relación de CI con el desempeño en las empresas[47] por lo que será difícil hacerlo igualmente en universidades. Todos los argumentos anteriores relacionados con las empresas podrían repetirse para las universidades; las principales diferencias serían los indicadores para medir el desempeño, ya que la mayoría de ellos serían diferentes.

\section{B. La Responsabilidad Social Corporativa}

Tanto las empresas como las universidades tienen que dar respuestas a las nuevas demandas sociales. Aunque, en los últimos años, ha habido una preocupación creciente sobre la insuficiencia de los informes en los negocios, no hay un acuerdo sobre lo que debe hacerse[48] La Responsabilidad Social Corporativa (RSC) se define por[49] como una nueva dimensión organizacional relacionada con su sostenibilidad y su impacto en los ámbitos económico, social y ambiental. Es un compromiso realizado por un grupo de personas para interactuar con la sociedad y contribuir a su mejoramiento.

Diferentes organizaciones nacionales e internacionales han emitido directrices, sobre información social y medioambiental, ya sea de manera obligatoria o voluntaria, tanto en Europa, Esta- dos Unidos y en Argentina. Una directriz internacional muy conocida es el Global Reporting Initiative (GRI), cuyo objetivo general es proporcionar información sobre la Responsabilidad Social Corporativa. La Comisión Europea emitió una recomendación sobre la medición y presentación de informes en los estados financieros de los gastos ambientales, los activos, pasivos y contingencias, que fue aprobada de manera obligatoria por varios miembros de la Unión Europea, incluida España[50]. Las Naciones Unidas, han puesto en marcha su programa 'Global Compact', con el fin de alinear de manera voluntaria empresas y otras entidades, universidades, entre ellas, con un conjunto de principios en materia de derechos humanos, laborales, medio ambiente y corrupción[30]; España está muy por delante en relación con el número de empresas y otras entidades que han firmado este compromiso[22].

Como parte de las actividades de la tercera misión, las universidades tienen que llegar a ser socialmente responsables, y demuestran que están respondiendo a las demandas de la sociedad.

\section{Las relaciones interorganizacionales}

Las alianzas y redes, como parte del capital relacional, es también una preocupación común para las diversas organizaciones. Del mismo modo que las empresas no pueden innovar de forma aislada, las universidades cada vez más confían en sus interrelaciones para innovar. La importancia de las relaciones exteriores de las empresas ha sido subrayada por OCDE en el Manual de Oslo, dedicado a la medición de la innovación[51].

Un problema importante relacionado con la mayoría de los intangibles incluidos en la categoría de capital relacional, son las alianzas y redes, en particular, se trata de identificar los indicadores cuantitativos para ellos y relacionar su existencia con el desempeño. Una vez más, esta dificultad es común tanto para empresas como universidades.

\section{Los Efectos de la divulgación del CI en los mercados de capital}

Las empresas compiten en los mercados de capital y las ventajas de la publicación de información sobre su capital intelectual han sido debidamente documentadas. Diferentes estudios demuestran cómo la divulgación reduce las 
asimetrías, aumenta la transparencia y por lo tanto disminuye el costo de capital[47].

El informe Ricardis sobre el capital intelectual para incrementar la investigación, el desarrollo y la innovación en las PYME[52] considera que hay dos tipos de efectos de los informes de CI en las empresas. La contribución a la gestión interna y complementa la información externa existente, lo que tiene un efecto en los mercados de capitales. Los mismos dos efectos podrían ser mencionados en relación con las universidades. Internamente ayuda a identificar sus principales activos y a mejorar el proceso de toma de decisiones. Externamente, las universidades compiten por becas y fondos de investigación para estudiantes y profesores.

\section{El Estado de LA INVESTIGACIÓN DE LOS INFORMES DE CAPITAL INTELECTUAL EN LAS UNIVERSIDADES}

La revisión de la literatura identificó una carencia de investigación para la divulgación del CI en universidades. Estas crean conocimiento mediante la investigación, en el largo plazo, actúan como guardianes de este conocimiento, lo trasmiten a los demás mediante procesos educativos, formación de nuevos investigadores y aplicaciones industriales[53]. En muchos países, tienen cada vez mayor autonomía en cuanto a su organización, gestión y asignación de presupuesto, lo que demanda nuevos sistemas de gestión y presentación de informes, y se enfrentan a varios retos nuevos causados por la iniciativa política , una economía y sociedad alteradas, así como nuevos modos de investigación[54]. Las universidades se enfrentan a la creciente demanda de los propietarios y ciudadanos por la transparencia en cuanto al uso de sus fondos, la divulgación de lo social y los resultados económicos de las universidades, y colaboran con otras instituciones de investigación , empresas privadas o entidades públicas, o incluso participan en redes de investigación internacional[54].

La comunidad académica, así como el público en general, suponen que el CI de una universidad debe estar llegando a los más altos niveles de excelencia y no requiere ningún tipo de intervención, pero la realidad contradice esta declaración debido a que las universidades de hoy en día son lentas para innovar[55]. Las universidades no tienen una estructura análoga a las empresas privadas, en consecuencia, no necesitan producir la clase de informes anuales prescritos en el derecho comercial, pero tienen que poner en práctica sistemas de contabilidad financiera[54].

La preparación de informes de CI para las universidades es más difícil que el de la industria, porque las universidades tienen una amplia gama de propósitos y objetivos que determinan su rendimiento[54]. La divulgación del CI para las universidades depende de la tarea de asignación de presupuestos, la forma explícita que define sus metas y estrategias organizacionales, la mayor autonomía y la competición extendida con otras organizaciones de investigación[54].

Las normas contables actuales limitan el reconocimiento de los activos intangibles[56]. Por esta razón, existen numerosos organismos reguladores internacionales, agencias e instituciones académicas que, conscientes de la dificultad de incorporar el CI en el balance, tienden a recomendar el desarrollo y la presentación del llamado informe de CI.

Los informes de CI contienen un conjunto de indicadores que contribuyen a mejorar la calidad de la información contable en las organizaciones. En esta línea, en el ámbito español, la comisión de expertos contables del ministerio de economía, en el 2002, recomienda la elaboración y publicación de un informe de CI siguiendo los lineamientos del proyecto Meritum[57], que consta de tres partes: una visión de la empresa, un resumen de las actividades y los recursos intangibles y un sistema de indicadores.

Teniendo en cuenta estas consideraciones, los autores consideran que la información no financiera es la más apropiada para suministrar información sobre los elementos intangibles de las universidades, evitando así la inclusión de criterios contables que podrían poner en peligro la calidad y fiabilidad de la información financiera. De esta manera, los sistemas de contabilidad de la universidad podrían mejorarse con la redacción y la presentación de una batería de indicadores. Este conjunto de indicadores mostraría la información más demandada por los diferentes stakeholders en relación con los recursos intangibles de la institución. La obligación de presentar un informe de CI en el sistema de educación superior sería un paso crucial hacia la nueva dirección de la universidad, lo que intenta un doble objetivo: identificar y medir los intangibles con fines de gestión, y proporcionar información útil a los interesados. 
A principios de 2001, el Instituto de Investigación en Electrónica y Telecomunicaciones (ETRI, por su sigla en inglés), una organización coreana sin ánimo de lucro, desarrolló una herramienta de gestión efectiva y estableció un sistema de gestión del conocimiento. Y, desde 2004 ETRI publica el informe de CI anualmente[58] La Universidad del País Vasco (UPV) desarrolló un estudio de caso de gestión del conocimiento científico-técnico, con el objeto de identificar los tipos de conocimiento relevantes para la gestión del proceso de $\mathrm{I}+\mathrm{D}+\mathrm{T}$, para la definición de indicadores adecuados[59].

A nivel europeo, en el año 2002 la Asociación Europea de Investigadores, Gerentes y Administradores, en colaboración con el Centro Europeo para la Gestión Estratégica de Universidades, lanzaron una iniciativa en relación con el CI en las instituciones de educación superior y organizaciones de investigación y tecnología, con el objetivo de sensibilizar y difundir la investigación sobre buenas prácticas en el ámbito de la gestión y presentación de informes de CI entre las universidades y organismos de investigación[60].

Una de las experiencias más interesantes en la presentación de información sobre CI es el de las universidades públicas de Austria, que están obligados a presentar informes de $\mathrm{CI}$ (conocidos como Wissensbilanz). La ley sobre universidades austriaca de 2002 - Ley sobre Estudios y Organización universitaria austriaca[61], en su artículo 13, estableció la obligación y el marco general para el desarrollo de este informe de CI. Según UG2002 (artículo 13, inciso 6), el informe de CI incluirá, al menos, los siguientes elementos: las actividades de la universidad, sus estrategias y objetivos sociales y voluntarios; el CI, dividido en capital humano, estructural y relacional; los procesos presentados en el contrato de desempeño, incluyendo resultados e impactos. El primer informe de CI debía publicarse en el 2005. Sin embargo, la orden ministerial (Ministerio Federal de Educación, Ciencia y Cultura de Austria,[61]) estableció la estructura del informe de CI de las universidades, y explicó la manera de presentar la información y los indicadores que deben ser incluidos obligatoriamente, no fue publicada hasta el 15 de febrero 2006. Así, las universidades austriacas realmente sólo se vieron obligadas a publicar el informe de $\mathrm{CI}$ a partir del 30 de abril de 2007.
En el informe Ricardis, el grupo de expertos de alto nivel motivó a las universidades a participar en los esfuerzos para desarrollar una cultura de informes de CI, como parte de una estrategia para la rendición de cuentas de las universidades a sus grupos de interés, mediante la mejora de la transparencia en su gobierno y sus estrategias y gestión de recursos[18]. Dicho informe recomendó el uso de tres categorías (Capital Humano, estructural y relacional) para el informe de CI de Universidades y Organizaciones de Investigación. Su consejo se basa en algunas experiencias internacionales. Una de las principales es la de los Centros de Investigación de Austria (ARC), la mayor organización de Investigación y Tecnología de Austria, que tiene una importante función de enlace entre la investigación básica en las universidades y la investigación aplicada realizada por las empresas. Ellos publicaron su primer informe de CI en el año 1999.

De otro lado, el Observatorio de Universidades Europeas (OEU) propuso la presentación del informe de capital intelectual llamado informe ICU[62] diseñado específicamente para las universidades y centros de investigación, con el objetivo de mejorar la transparencia y ayudar a la difusión homogénea de los indicadores de capital intelectual. El informe ICU propuesto consta de tres secciones fundamentales que describen el movimiento lógico desde la estrategia interna (diseño de la visión y los objetivos de una institución), la gestión hasta un sistema de indicadores (OEU)[63]: (a) la visión de la institución; (b) los recursos y actividades intangibles; (c) El sistema de métricas.

De igual manera, el Programa de Indicadores de Capital Intelectual, PCI Project (2000-2003) aplicado a universidades y centros de investigación en el área de Madrid[64] una iniciativa liderada por los miembros de la Asociación Europea de Administradores y Gerentes de Investigación (EARMA) llamada capital intelectual en organizaciones de investigación y de Educación Superior (HEROs) [53] el caso de la Universidad de Poznan, en Polonia, donde Fazlagic[55] presenta un informe de CI basado en la metodología propuesta por la Danish Agency for Trade and Industry[65], en la que el CI se presenta en forma de recursos, actividades y resultados.

Otras experiencias más recientes, Bezhani[24] realiza un trabajo cuyo propósito es analizar las 
prácticas sobre reportes de CI para el logro de los siguientes objetivos:

- Evaluar la naturaleza y la actitud de las universidades del Reino Unido hacia la importancia de la divulgación voluntaria del CI;

- Evaluar la influencia del desempeño de dichas universidades en la divulgación del CI; y su opinión sobre la divulgación obligatoria del CI;

- Sugerir recomendaciones para la divulgación del CI y tener una base para estudios comparativos con otros proveedores de educación profesional en el Reino Unido o con universidades de otros países.

La metodología utilizada a una muestra de 30 universidades, considera una combinación de métodos de investigación cualitativa y cuantitativa e identificó el uso del análisis de contenido y el cuestionario como los más apropiados. Los datos primarios del cuestionario complementados con los datos secundarios de los informes anuales de las universidades, son la fuente más popular de obtención de datos de muchos estudios de divulgación del CI. El análisis de contenido, se fundamentó en la clasificación de la información de CI en categorías y sub-categorías de acuerdo al esquema de codificación predefinida en 39 indicadores agrupados de manera obligatoria en ocho categorías, usada por Leitner[55], para el informe obligatorio de universidades Austriacas: el capital humano; el capital relacional; El capital estructural; la investigación; la educación; la comercialización; la transferencia de conocimiento al público, y los servicios.

El estudio de Elena y Warden[66] confirma la aplicabilidad de un informe de CI en las instituciones de educación superior. Para ello, se llevaron a cabo 30 entrevistas en dos universidades españolas diferentes (la Universidad Autónoma de Madrid y la Universidad Pablo de Olavide). Sus resultados muestran que los enfoques de CI son útiles para la comunicación tanto interna como externa. Al aplicarlos, los efectos positivos más importantes o beneficios que las universidades pueden obtener son: la mejora de la gestión interna, la transparencia y las relaciones con sus grupos de interés.

Más recientemente, la Universidad Nacional de Colombia publicó el texto capacidades de investi- gación 2000-2011 desde la perspectiva del capital intelectual (humano, relacional y estructural), y está próxima a implementar un sistema de 38 indicadores para medir sus capacidades en ciencia y tecnología. De estos ocho están enfocados en su capital humano, nueve, en su capital relacional y veinte, en su capital estructural[67].

En la mayoría de los países no existe ninguna obligación o recomendación a las universidades para medir y presentar información sobre su CI. Con el fin de resolver esta situación, al menos en España, Ramírez y Gordillo[68], desarrollaron una propuesta de modelo de medición del CI para las universidades españolas. Dicho propósito se logró mediante los siguientes objetivos:

- Analizar las iniciativas más importantes en la medición y presentación de informes de CI en las instituciones de educación superior.

- Identificar los elementos intangibles claves desde la perspectiva de los miembros de los consejos sociales de las universidades públicas españolas.

- Elaboración de una batería de indicadores para medir los elementos intangibles clave previamente identificados.

De un conjunto de 42 elementos intangibles propuestos inicialmente de acuerdo a las características de las universidades españolas mediante un cuestionario, los resultados obtenidos ponen de manifiesto los siguientes 13 elementos intangibles considerados como clave en las universidades españolas, las calificaciones académicas y profesionales del personal docente e investigador, la movilidad de profesores e investigadores, la productividad científica y competencias y capacidades de enseñanza (capital humano); esfuerzo en la mejora e innovación, la propiedad intelectual y la calidad de la gestión (capital estructural) ; así como la empleabilidad de los graduados, la eficiencia de la enseñanza de posgrado, satisfacción de los estudiantes, las relaciones con las empresas, la imagen de la universidad y la colaboración con otras universidades (capital relacional).

En Tabla II se sintetiza el Estado de la investigación sobre la divulgación del CI Universitario. 
Tabla II. Estado de la investigación sobre divulgación del Cl Universitario.

\begin{tabular}{|c|c|c|c|c|}
\hline $\begin{array}{l}\text { Nombre de la } \\
\text { investigación }\end{array}$ & Autores & Año & Origen & Aspectos considerados \\
\hline Proyecto Meritum & $\begin{array}{l}\text { Cañibano, L., Sánchez, P., } \\
\text { García-Ayuso, M. } \\
\text {,Chaminade, C. [57] }\end{array}$ & 2002 & España & $\begin{array}{l}\text { Visión de la empresa, resumen de las actividades y los } \\
\text { recursos intangibles y un sistema de indicadores }\end{array}$ \\
\hline Proyecto PCI & Comunidad de Madrid [64] & 2002 & España & $\begin{array}{l}\text { La estructura de CI usada en las empresas se puede } \\
\text { aplicar en las universidades y centros de investigación }\end{array}$ \\
\hline $\begin{array}{l}\text { Gestión del conocimiento } \\
\text { científico-técnico en la } \\
\text { universidad del país } \\
\text { Vasco }\end{array}$ & $\begin{array}{l}\text { Rodríguez, A., Charterina, J., } \\
\text { Hartmann, P. [59] }\end{array}$ & 2003 & España & $\begin{array}{l}\text { Identificación de tipos de conocimiento relevantes } \\
\text { para la gestión del proceso de } \mathrm{I}+\mathrm{D}+\mathrm{T} \text { para la } \\
\text { definición de indicadores adecuados }\end{array}$ \\
\hline $\begin{array}{l}\text { Sistema de Gestión de } \\
\text { conocimiento ETRI }\end{array}$ & $\begin{array}{l}\text { Electronics and } \\
\text { Telecommunications } \\
\text { Research Institute [58] }\end{array}$ & 2004 & Corea & Reporte de capital intelectual \\
\hline $\begin{array}{l}\text { Capital intelectual en IES } \\
\text { Y Organizaciones de } \\
\text { Investigación y } \\
\text { Tecnología. }\end{array}$ & Leitner [60] & 2005 & Europa & Gestión y presentación de informes de CI \\
\hline $\begin{array}{l}\text { Medición del CI en la } \\
\text { Universidad de Poznan }\end{array}$ & Fazlagic [55] & 2005 & Polonia & Recursos, actividades y resultados \\
\hline Reporte de capital ARC & $\begin{array}{l}\text { Ministerio Federal de } \\
\text { Educación, Ciencia y } \\
\text { Cultura de Austria [61] }\end{array}$ & 2006 & Austria & $\begin{array}{l}\text { Actividades universitarias, estrategias y objetivos } \\
\text { sociales; el CI, dividido en capital humano, estructural } \\
\text { y relacional; los procesos, incluyendo resultados e } \\
\text { impactos }\end{array}$ \\
\hline Reporte Ricardis & $\begin{array}{l}\text { Dirección General de } \\
\text { Investigación y Desarrollo } \\
\text { Tecnológico de la Comisión } \\
\text { Europea [64] } \\
\end{array}$ & 2006 & Europa & $\begin{array}{l}\text { El uso de tres categorías (Capital Humano, estructural } \\
\text { y relacional) para el informe de CI de Universidades y } \\
\text { Organizaciones de Investigación. }\end{array}$ \\
\hline Informe ICU & Sanchez et al [62] & 2006 & Europa & $\begin{array}{l}\text { la visión de la institución; los recursos y actividades } \\
\text { intangibles; El sistema de métricas. }\end{array}$ \\
\hline $\begin{array}{l}\text { Reporte de CI en } \\
\text { universidades del Reino } \\
\text { Unido }\end{array}$ & Bezhani [24] & 2010 & Reino Unido & $\begin{array}{l}\text { Evaluación de la influencia del desempeño en la } \\
\text { divulgación del CI }\end{array}$ \\
\hline $\begin{array}{l}\text { Aplicabilidad del CI en } \\
\text { Universidades Autónoma } \\
\text { de Madrid y Pablo } \\
\text { Olavide }\end{array}$ & Elena y Warden [66] & 2011 & España & $\begin{array}{l}\text { Gestión interna, transparencia y relaciones con grupos } \\
\text { de interés }\end{array}$ \\
\hline $\begin{array}{l}\text { Modelo Intellectus } \\
\text { versión actualizada }\end{array}$ & Bueno et al [28] & 2011 & España & $\begin{array}{l}\text { Cuatro categorías: humano, estructural, relacional, } \\
\text { emprendimiento e innovación }\end{array}$ \\
\hline $\begin{array}{l}\text { Capacidades de } \\
\text { investigación en la UNAL } \\
\text { desde la perspectiva del } \\
\text { CI }\end{array}$ & Sánchez [67] & 2012 & Colombia & $\begin{array}{l}\text { Sistema de } 38 \text { indicadores para medir sus capacidades } \\
\text { en ciencia y tecnología }\end{array}$ \\
\hline $\begin{array}{l}\text { Modelo de medición del } \\
\text { CI para las universidades } \\
\text { españolas }\end{array}$ & Ramírez y Gordillo [68] & 2014 & España & $\begin{array}{l}\text { Elaboración de una batería de } 13 \text { indicadores clave } \\
\text { para medir los elementos intangibles }\end{array}$ \\
\hline
\end{tabular}

Fuente: elaboración propia 


\section{CONCLUSIONES}

Las iniciativas de divulgación del CI de las universidades en América Latina son escasas o inexistentes.

Después de veinte años de la publicación del artículo de Stewart [69], de manera justa puede afirmarse que el progreso ha sido lento. La evidencia de esto es que el número de universidades que utilizan metodologías de capital intelectual en todo el mundo es muy baja, y quienes divulgan los informes de CI es aún más baja.

Si las universidades deben divulgar sus recursos de capital intelectual, entonces la necesidad de un acuerdo sobre las definiciones de los términos, evitando lagunas o solapamientos en su significado, es aún más fuerte.

La consideración del capital emprendimiento e innovación en la gestión universitaria, facilitará el paso al paradigma de 'universidades emprendedoras', que suponen cambios globales en la cultura, organización y formas de funcionamiento, en los métodos de gestión, y relación de las universidades como respuesta a las presiones del entorno en que trabajan.

La visión microeconómica de la empresa, que concibe que esta se ??mide mejor por alguna medida de los recursos productivos utilizados, ha influenciado el desarrollo del capital intelectual en su perspectiva estratégica, al considerar los activos intangibles clave para el logro de la estrategia; en su perspectiva de gestión empresarial del CI, que permite la creación de valor; y en su perspectiva contable, que permite medir y presentar los componentes del CI.

Las técnicas Scordcard (SC) como "academia scorecard" han mostrado ser un enfoque alternativo que permite enriquecer el control de la gestión de los intangibles de las universidades.

La carencia de investigación para la divulgación del CI en universidades no ha facilitado una mayor velocidad de innovación en ellas, tampoco una gestión adecuada, ni una mejor transparencia, ni un mejor relacionamiento con los diversos grupos de interés.
La combinación de métodos cuantitativos de investigación como el cuestionario, y cualitativos como el análisis de contenido, son apropiados para la recolección de datos primarios y secundarios de los estudios de divulgación del CI.

Conflicto de intereses: El autor declara no tener ningún conflicto de intereses

Agradecimientos: Los resultados de investigación presentados en este artículo hacen parte del proyecto Gestión del Capital Intelectual en universidades financiado por la Funlam.

\section{REFERENCIAS}

[1] Y. Ramirez, A. Tejada, M. Manzaneque, «The value of disclosing intellectual capital in Spanish universities: A new challenge of our days», Journal of Organizational Change Management, 29, 2,176 - 198. 2016.

[2] J. Guthrie \& V. Murthy, Past, present and possible future developments in human capital accounting: a tribute to Jan-Erik Grojer. Journal of Human Resource Costing \& Accounting, 13,2, 125-142. 2009.

[3] E. Chamberlin, The Theory of Monopolistic Competition, Harvard University Press, Cambridge, MA. 1933.

[4] E. Penrose, The Theory of Growth of the Firm, Blackwell, Oxford. 1959.

[5] J. Schumpeter, The Theory of Economic Development, 1912, Harvard University Press, Cambridge, MA, trans Redvers Opie (first published 1912). 1934.

[6] J. Robinson, The Economics of Imperfect Competition, Macmillan, London. 1933.

[7] G. Day, "The capabilities of market-driven organisations", Journal of Marketing, 58, 37-52. 1994.

[8] R. Hall, "The strategic analysis of intangible resources", Strategic Management Journal, 13, 2, 135-44. 1992.

[9] R. Amit and P. Schoemaker, "Strategic assets and organisational rent", Strategic Management Journal, 14,1, 33-46. 1993.

[10] J. Barney, "Strategic factor markets: expectations, luck and business strategy", Management Science, 32, 10, 1231-41. 1986.

[11] J. Barney, "Firm resources and sustained competitive advantage", Journal of Management, 17, 1, 99-120. 1991. 
[12] D. Lei, M. Hitt and R. Bettis, "Dynamic core competences through meta-learning and strategic context", Journal of Management, 22, 4, 549-69. 1996.

[13] P. Schoemaker, How to link strategic vision to core capabilities, Sloan Management Review, 34, 67-81. 1992.

[14] S. Chatterjee and B. Wernerfelt, "The link between resources and type of diversification: theory and evidence", Strategic Management Journal, 12, 1, 3348. 1991.

[15] C. Markides and P. Williamson, "Corporate diversification and organisational structure: a resource-based view", Academy of Management Journal, 39, 2, 340-67. 1996.

[16] C. Prahalad and G. Hamel, “The core competence of the corporation", Harvard Business Review, 68, 79-91. 1990.

[17] J. Robins and M. Wiersema, "A resource-based approach to the multi-business firm: Empirical analysis of portfolio interrelationships and corporate financial Performance", Strategic Management Journal, 16, 277-99. 1995.

[18] European Commission, "Ricardis: reporting intellectual capital to augment research, development and innovation in SMEs. Report to the Commission of the High Level Expert Group on Ricardis", available at: http:/ / ec.europa.eu/investin-research/pdf/ download_en/ 2006-2977_ web1.pdf (accessed 29 November 2014). 2006.

[19] K. H. Leitner, "Intellectual capital reporting for universities: conceptual background and application for Austrian Universities", Research Evaluation, 13, 2, 129-140. 2004.

[20] S. Elena, "Governing the university of the 21st century: intellectual capital as a tool for strategic management. Lessons from the European experience" ${ }^{\prime \prime}, \mathrm{PhD}$ dissertation, Universidad Autónoma de Madrid, Madrid. 2007.

[21] Y. Ramírez, C. Lorduy and J. A. Rojas, "Intellectual capital management in Spanish Universities", Journal of Intellectual Capital, 8, 4, 732-748. 2007.

[22] L. Cañibano y P. Sánchez, "Intellectual capital management and reporting in universities and research institutions", Estudios de Economía Aplicada, 26, 2, 7-26. 2008.

[23] P. Sánchez, S. Elena and R. Castrillo, “Intellectual capital dynamics in universities: a reporting model", Journal of Intellectual Capital, 10, 2, 307324. 2009.

[24] I. Bezhani, Intellectual capital reporting at UK universities. Journal of Intellectual Capital, 11,2, 179-207. 2010.
[25] V. Bodnar, T. Harangozo, T. Tirnitz, E. Revesz and G. Kovats, "Managing intellectual capital in Hungarian Universities - the case of Corvinus University of Budapest", Proceedings of the 2nd European Conference on Intellectual Capital in Lisbon, 89-126. 2010.

[26] C. Casanueva and A. Gallego, "Social capital and individual innovativeness in university research networks", Innovation: Management, Policy \& Practice,12, 1, 105-117. 2010.

[27] G. Secundo, A. Margheritam, G. Elia and G. Passiante, "Intangible assets in higher education and research: mission, performance or both?", Journal of Intellectual Capital, 11, 2, 140-157. 2010.

[28] E. Bueno et al. Modelo Intellectus de medición, gestión e información del capital intelectual. Nueva versión actualizada. IADE, 9:1-80. 2011.

[29] D. H. Luthy, Intellectual capital and its measurement, Proceedings of the asian pacific interdisciplinary research in accounting conference (APIRA), Japan, (Available at: www.bus.osakacu.ac.jp/apira98/archives/htmls/25.htm).1998.

[30] O. F. Williams, "The UN global compact: the challenge and the promise", Business Ethics Quarterly, 14. 2004.

[31] L. Gogan, A. Draghici, A Model to evaluate the intellectual capital. Procedia Technology 9, 867875. 2013.

[32] J. Jurczak, Intelelctual capital measurement methods, Economics and Organization of Enterprise, 1,1, 37-45. 2008.

[33] B. Lev and S. Zambon, Intangibles and intellectual capital: an introduction to a special issue, European Accounting Review, 12, 4, 597-603. 2003.

[34] J. Mouritsen, Overview intellectual capital and the capital market: the circulability of intellectual capital, Accounting, Auditing \& Accountability Journal, 16, 1, 18-30. 2003.

[35] M. Pupius et al. Linking the EFQM Excellence Model to other management models and tools. Sheffield, Sheffield Hallam University. 2003.

[36] S. Johnson, EFQM and Balanced Scorecard for improving organisational performance. Aresearch report. Available at: http:/ / www.som.cranfield. ac.uk/som/cbp/pma/Balanced \% 20Scorecard \% 20and\%20EFQM.pdf. 2003.

[37] J. Cullen, J. Joyce, T. Hassall, M. Broadbent, “Quality in higher education: from monitoring to management", Quality Assurance in Education, 11, 1, 514. 2003

[38] H. Rohm, Improve public sector results with a Balanced Scorecard: Nine steps to success. The

Rev. Ingeniería, Matemáticas y Ciencias de la Información Vol. 6 / Núm. 11 / enero - junio de 2019; pág. 131-144 
Balanced Scorecard Institute. Available at: http:/ / www.balancedscorecard.org. 2003.

[39] University of California San Diego: A University Business Model Based on the Balanced Scorecard. Mexico/USA University Discussion, July, 27. UC San Diego. http:/ / www-vcba.ucsd.edu/mexicousa/sld026.htm. 1999.

[40] JR. O'Neil y F. Y. Harold, “Designing and Implementing an Academic Scorecard", U.S. News \& World Report, 31, 6, 30-32. 1999.

[41] Universitat Pompeu Fabra,Cuadro de mando integral. 1999-2000. Disponible en: http:/ / www.upf. es/grec/cast/coneixer/1/qci.htm. 2000.

[42] Universitat Jaume I, Sistema de dirección estratégica. Documento Base de Planificación. Available at: http:/ / www.uji.es. 2000.

[43] M. Porporato, N. García, "Análisis de las limitaciones del Cuadro de Mando Integral: Revisión de la literatura e implicaciones para la práctica", Revista Iberoamericana de Contabilidad de Gestión, 9, 13-32. 2007.

[44] D. Foray, The Economics of Knowledge, MIT, Cambridge, Massachusetts. 2004.

[45] M. Augier and D. J. Teece, "An economic perspective on Intellectual Capital", in Marr, B. (Ed.), 3-27. 2005.

[46] B. Lev, Intangibles: Management, Measurement and Reporting, The Brookings Institution, Washington D.C. 2001.

[47] L. Cañibano and M. Sánchez, "Measurement, management and reporting on intangibles. State of the art", Cañibano, L. Sanchez, M.P. (Eds.), Readings on Intangibles and Intellectual Capital, AECA, 81-113. 2004.

[48] Institute of Chartered Accountants in England and Wales: Information for better markets. New reporting models for business. 2005.

[49] AECA: Marco Conceptual de la Responsabilidad Social Corporativa, Documentos AECA, Madrid. 2004.

[50] ICAC: "Normas para el reconocimiento, valoración e información de los aspectos medioambientales en las cuentas Anuales", Boletín ICAC, 49, 29-34. 2002.

[51] OECD: Oslo Manual: Guidelines for Collecting and Interpreting Innovation, 3rd Edition, OECD Publications, París. 2005.

[52] Meritum: Guidelines for Managing and Reporting on Intangibles (Intellectual Capital Statements), Cañibano, L.; Sánchez, P.; García-Ayuso, M.; Chaminade, C. (Eds.), Vodafone Foundation, Madrid. 2002.
[53] C. Warden, "Managing and reporting intellectual capital: new strategic challenges for HEROs", IP Helpdesk Bulletin, 8, April-May, available at: www.ipr-helpdesk.org/ newsletter/8/pdf/EN/ N08_EN.pdf (accessed 30 October 2006). 2003.

[54] K. H. Leitner, "Intellectual capital reporting for universities: conceptual background and application within the reorganisation of Austrian universities", paper presented at The Transparent Enterprise, The Value of Intangibles Conference, Autonomous University of Madrid Ministry of Economy, Madrid. 2002.

[55] A. Fazlagic, "Measuring the intellectual capital of a university", paper presented at the Conference on Trends in the Management of Human Resources in Higher Education, OECD, Paris, available at: www.oecd.org/dataoecd/56/16/35322785.pdf (accessed 28 March 2014). 2005.

[56] L. Cañibano, A. Gisbert, E. García-Meca and B. García-Osma, Los intangibles en la regulación contable, Documento AECA \& Instituto Análisis Intangibles, Madrid. 2008.

[57] L. Cañibano, P. Sánchez, M. García-Ayuso and C. Chaminade, Directrices para la Gestión y Difusión de Información sobre Intangibles. Informe de Capital Intelectual, Proyecto Meritum, Vodafone Fundación, Madrid. 2002.

[58] Electronics and Telecommunications Research Institute, "Intellectual capital report", available at: www.etri.er.kr (accessed 12 march 2014). 2005.

[59] A. Rodríguez, J. Charterina, P. Hartmann, Vinculaciones entre tipos de conocimiento relevantes para la gestión de la I+D en la universidad: análisis de un caso. Revista Economía y Empresa, 19, 48, 145-169. 2003.

[60] K. H. Leitner, "Managing and reporting intangible assets in research technology organisations", R\&D management, 35, 2, 125-136. 2005.

[61] Federal Ministry of Education, Science and Culture of Austria, Verordnung ueber die Wiessenbilanz (Wissensbilanz-Verordnung-WBV), BGB1, II Nr.63/2006, available at: www.bmbwk.gv.at/ universiteeten/recht/gesetze/wbv/wbv.xml. 2006.

[62] P. Sánchez, S. Elena and R. Castrillo, "The Intellectual Capital Report of Universities. Guidelines for disclosing IC information", in Observatory of the European University (Ed.), PRIME-OEU Methodological Guide, 223-251. 2006.

[63] Observatory of European of University (OEU): Methodological Guide, Final Report of the Observatory of the European University, PRIME Project, available at: www.prime-noe.org (accessed 14 February 2014). 2006. 
[64] Comunidad de Madrid: "Capital Intelectual y Producción Científica", Dirección General de Investigación, Consejería de Educación, Comunidad de Madrid, Madrid, Spain. 2002

[65] Danish Agency for Trade and Industry. A guide for Intellectual Capital- A key to Knowledge Management. Copenhagen. 2000.

[66] S. Elena and C. Warden, "Visualising the hidden value of higher education institutions: how to manage intangibles in knowledge-intensive organisations", in Vallejo, B., Rodríguez, A. and Arregui, G. (Eds), Identifying, Measuring, and Valuing Knowledge-Based Intangible Assets, New perspectives, IGI Global, New York, NY, p. 177207. 2011.
[67] J. Sánchez, Capacidades de investigación en la Universidad Nacional de Colombia 2000-2011: una aproximación desde el capital intelectual. Bogotá, 216p. 2012.

[68] Y. Ramírez, S. Gordillo, «Recognition and measurement of intellectual capital in Spanish universities», Journal of Intellectual Capital, 15, 1, 173-188. 2014.

[69] T. Stewart, "Your company's most valuable asset: intellectual capital", Fortune Magazine, October. 1994. 